\title{
Case study of the development of polar stratospheric clouds using bistatic imaging
}

\author{
C.-F. Enell ${ }^{1}$, U. Brändström ${ }^{1}$, B. Gustavsson ${ }^{2}$, S. Kirkwood ${ }^{1}$, K. Stebel ${ }^{3}$, and A. Steen ${ }^{4}$ \\ ${ }^{1}$ Swedish Institute of Space Physics, Kiruna, Sweden \\ ${ }^{2}$ National Institute of Polar Research, Tokyo, Japan \\ ${ }^{3}$ Norwegian Institute of Air Research, Troms $\emptyset$, Norway \\ ${ }^{4}$ RemSpace Group, Övlandehult, Sweden
}

Received: 13 September 2002 - Revised: 31 January 2003 - Accepted: 21 February 2003

\begin{abstract}
The formation of polar stratospheric clouds (PSCs) is closely related to wave activity on different scales since waves propagating into the stratosphere perturb the temperature profile. We present here a case study of the development of visible PSCs (mother-of-pearl clouds), appearing at the polar vortex edge on 9 January 1997, undertaken by means of ground-based cameras. It is shown that the presence of stratospheric clouds may be detected semiautomatically and that short-term dynamics such as altitude variations can be tracked in three dimensions. The PSC field showed distinct features separated by approximately $20 \mathrm{~km}$, which implies wave-induced temperature variations on that scale. The wave-induced characteristics were further emphasised by the fact that the PSCs moved within a sloping spatial surface. The appearance of visible mother-of-pearl clouds seems to be related to leewave-induced cooling of air masses, where the synoptic temperature has been close to (but not necessarily below) the threshold temperatures for PSC condensation.
\end{abstract}

Key words. Atmospheric composition and structure (aerosols and particles) - Meteorology and atmospheric dynamics (middle atmosphere dynamics; instruments and techniques)

\section{Introduction}

Polar stratospheric clouds (PSCs) play a very important role in atmospheric chemistry. The formation of PSCs involves condensation of water, $\mathrm{H}_{2} \mathrm{SO}_{4}$ and $\mathrm{HNO}_{3}$, and freezing of nitric acid trihydrate (NAT) and water (Peter, 1997). Low stratospheric temperatures are required; the NAT condensation temperature $T_{\mathrm{NAT}}$ (Table 1) is an often used threshold value (Hanson and Mauersberger, 1988). In the orographically disturbed Arctic polar vortex the synoptic-scale temperature is often higher and PSC formation takes place where local cooling by mountain-induced waves occurs (Dörnbrack

Correspondence to: C.-F. Enell (carl-fredrik.enell@irf.se) et al., 2001, and references therein). This is often the case when the vortex edge is located over the Scandinavian mountain ridge, close to our site of observation in Kiruna $\left(67^{\circ} \mathrm{N}, 20^{\circ} \mathrm{E}\right)$, and the prevailing tropospheric wind direction is from the northwest. Therefore PSCs observed here may show characteristic dynamic features on a scale which is not resolved in the synoptic-scale meteorological or chemistry/transport models generally used for assimilation of atmospheric data (including estimation of PSC area). Processes at this scale are inherently non-asymptotic (none of the force terms in the dynamic equations are fully negligible, as implied by the definition of mesoscale) and thus difficult to parameterise. We apply here a mapping of bistatic images in a case study to track the development of a PSC field of this kind.

This article covers three topics: a description of the bistatic imaging technique and an algorithm for automatic bistatic altitude determination, a test of the algorithm with a simplistic model and the images from the case study, and manual triangulations for the same case. The results are compared and related to the dynamic conditions. This case supports the idea that the visual appearance of "mother-of-pearl" colours appears to be the result of the rapid leewave cooling.

\section{Methods}

The generally applied methods for PSC studies fall into the category of one-point optical observations. In satelliteborne extinction measurements, wave-induced PSCs can be regarded as single pixel events, whereas semi-Lagrangian flights with airborne lidars (applied, for example by Godin et al., 1994; Carslaw et al., 1998a) track the microphysical development of single air parcels.

Ground-based lidars and cameras both provide time resolution on the order of one minute, but areas of application are different. Imaging gives the opportunity to study the large-scale development of a PSC field and its small-scale 


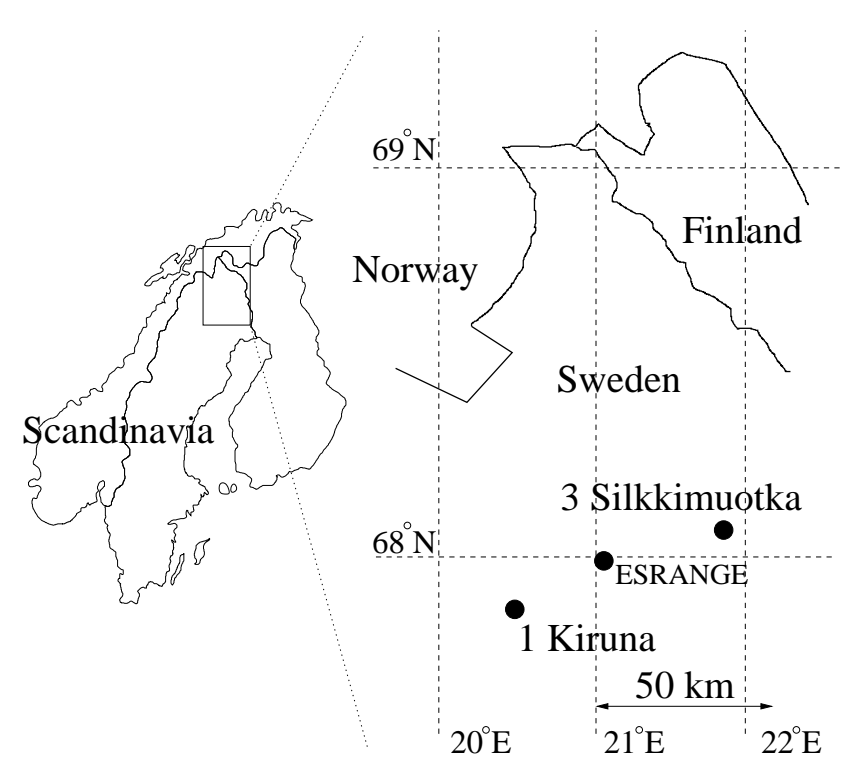

Fig. 1. Map of northernmost Sweden, showing the locations of ALIS station 1 (Kiruna, $67^{\circ} 50^{\prime} 27^{\prime \prime} \mathrm{N}, 20^{\circ} 24^{\prime} 40^{\prime \prime} \mathrm{E}$ ), ALIS station 3 (Silkkimuotka, $68^{\circ} 1^{\prime} 47^{\prime \prime} \mathrm{N}, 21^{\circ} 41^{\prime} 13^{\prime \prime} \mathrm{E}$ ), and ESRANGE.

features in relation to wave activity, whereas the lidars are better suited to studies of microphysics and composition.

\subsection{Imaging}

For the purpose of PSC imaging, ALIS, the Auroral Large Imaging System (Brändström and Steen, 1994), was applied in this study. ALIS consists of six remote-controlled stations, each equipped with a CCD imager. The two ALIS stations applied in this work are shown in Fig. 1. The baseline distance between the stations is adapted for auroral tomography, i.e. imaging of volumes above $100 \mathrm{~km}$. However under suitable conditions, PSCs located between the stations can also be imaged; see also Steen et al. (1998).

The ALIS cameras have a field-of-view of $54^{\circ}$ and are equipped with telecentric lens systems with one open (whitelight) position and 5 different narrow-band interference filters $(\mathrm{FWHM} \approx 40 \AA)$. In this study, the white-light and $5590 \AA$ positions have been used. The image detectors used are cooled CCDs, with $1024 \times 1024$ imaging pixels divided into four $512 \times 512$-pixel quadrants for fast readout.

\subsubsection{Image processing}

A few corrections must be applied to each image. The differences between sensitivity and CCD bias levels in the four quadrants are compensated by using the values of covered so-called overscan pixels on the CCD. Thereafter, a flat-field correction is applied. This compensates for the image radiance falloff towards the corners of the images caused by the decrease in the effective front-lens area,and projected pixel and object areas, as the angle between the pixel and the optical axis increases. We finally subtract a background (approximation of non-PSC scattering) obtained by fitting a smooth

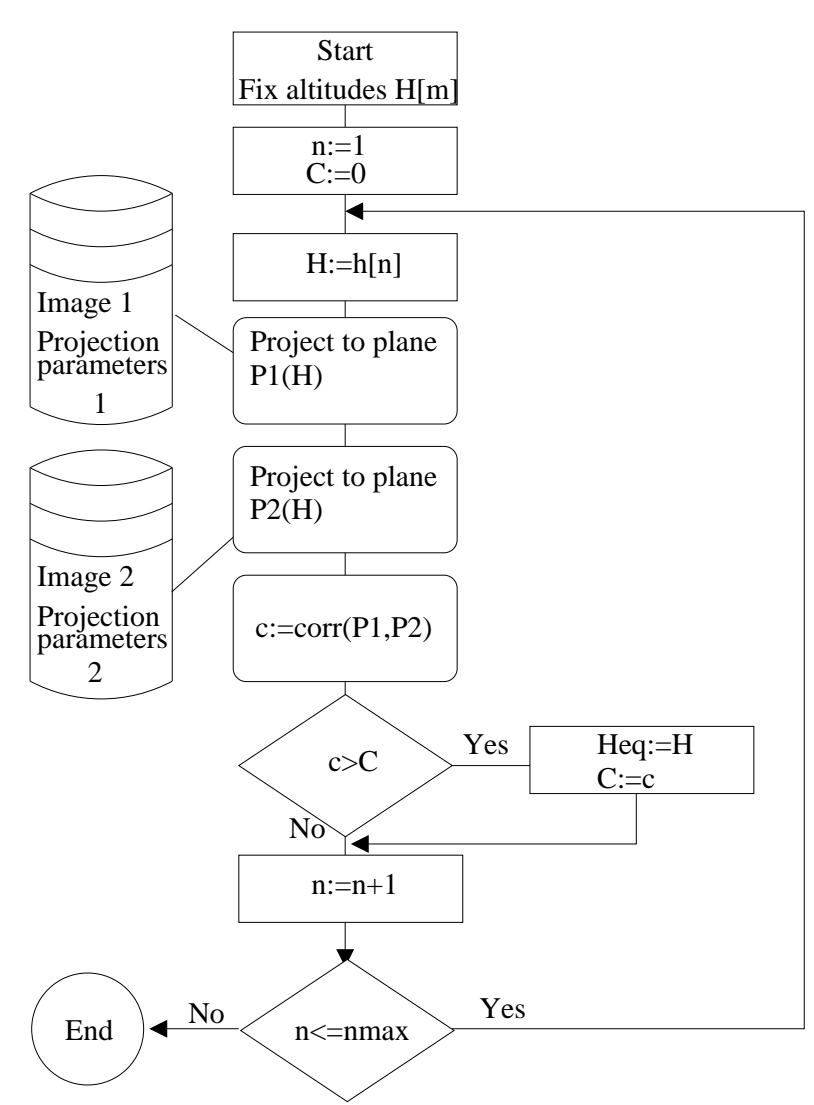

Fig. 2. Flowchart of the algorithm for automatic determination of the equivalent altitude of best matching, $H_{\text {eq }}$. The designations Image 1 and Image 2 can refer to either full images or subblocks/regions of interest. In the present version the altitudes of mapping are fixed.

surface to the minimum pixel values over fixed $x \times y$-pixel image sub-blocks. As will be seen in Fig. 14 below, this procedure renders the PSCs visible against a dark background.

\subsection{Triangulation}

The projection $(\varphi, \theta)=f(u, v)$, where $(\varphi, \theta)$ is the line-ofsight direction from a station and $(u, v)$ is the corresponding pixel in its image, can be accurately determined by virtue of star calibration (Gustavsson, 2000). Every pixel centre can therefore be mapped to a unique line of sight. Using bistatic imaging, the intersection of lines-of-sight from pixels corresponding to the same physical feature in pairs of images will, in principle, determine the geographic coordinates of the feature. In practice there will always be an error in the identification of corresponding pixels, so the lines-of-sight will not intersect. The physical coordinates are therefore found by minimising the separations between the lines of sight as functions of the pixel positions.

\subsection{Spatial correlation for approximate altitude mapping}

Triangulation of (stratospheric and other) clouds and the study of their scattering properties is not a trivial problem. 


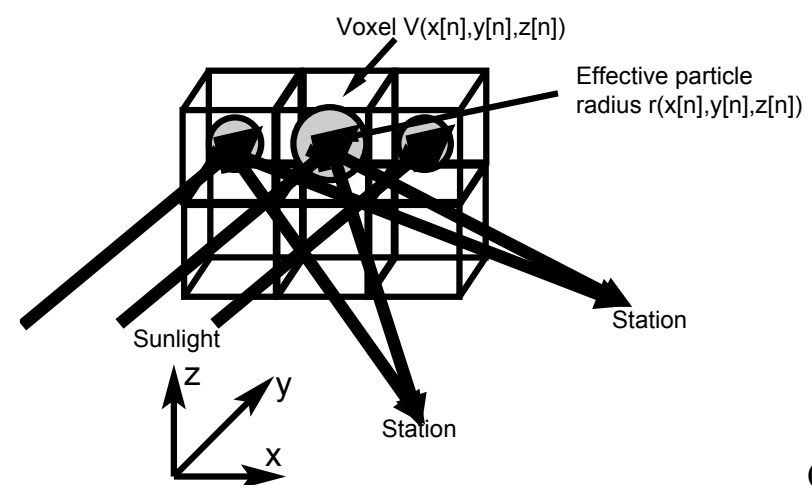

(a)

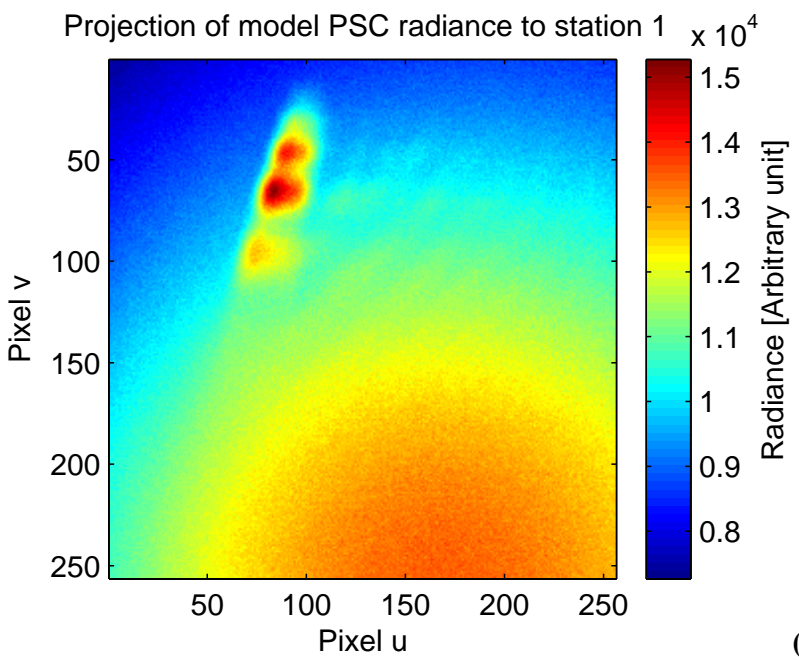

(b)

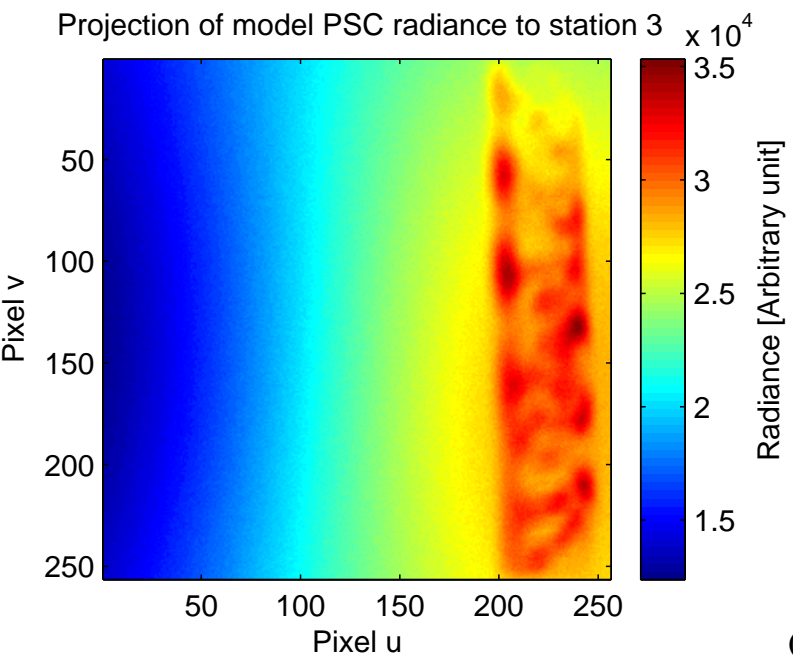

(c)

Fig. 3. The principle of the single-scattering model type case for altitude matching. Upper panel (a): Outline of the model volume. Lower panels: Resulting projections to stations 1 (b) and 3 (c) by scattering from the model volumes at $5590 \pm 40 \AA$, assuming sunset in the south-southwest.

Clouds have no well-defined surfaces, which has led to the adoption of a principle of defining "cloud surfaces" as features showing high spatial correlation when viewed from separate directions. The underlying assumption is that cloud pixels in the separate images are highly spatially correlated,

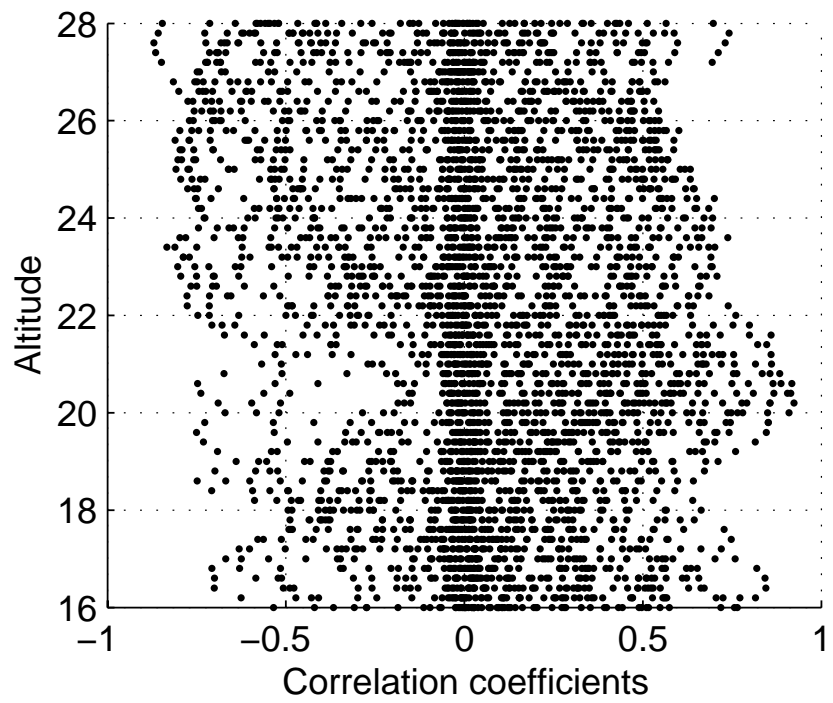

Fig. 4. Correlation coefficients for 128x128-pixel image sub-blocks at 61 projected altitudes $(16-28 \mathrm{~km})$, showing the presence of an object at $20 \mathrm{~km}$ in the modelled pair of test images.

whereas the background is noisy.

Figure 2 shows the principle behind the algorithm. The full images or regions of interest are projected to the lineof-sight directions and scaled to physical coordinates on a series of (arbitrary) planes of a fixed altitude. For each plane the correlation of the projected pixels is calculated and the altitude plane of maximal pixel correlation is chosen as the equivalent PSC altitude.

\section{Modelled test case}

The performance of the automatic algorithm was tested with a model case outlined in Fig. 3a. For volume elements (voxels) of constant particle radius, assumed to be located at fixed positions with respect to stations 1 and 3, the singlescattering phase functions for the scattering angles defined by the incident sunlight and the directions towards the stations were calculated with the Bohren and Huffman (1983) Mie code. For simplicity a somewhat unrealistic monodisperse radial distribution of the form

$r=r_{0} \exp \left[\left(\frac{x-x_{c}}{s_{x}}\right)^{2}+\left(\frac{y-y_{c}}{s_{y}}\right)^{2}+\left(\frac{z-z_{c}}{s_{z}}\right)^{2}\right]$

was applied, with coefficients appropriately chosen to produce radii in the two orders of magnitude between 0.1 and $10 \mu \mathrm{m}$ over the model volume. This range was chosen to include the range of sizes expected in PSCs and also smaller and larger sizes. Particles much smaller or larger than the wavelength should produce Rayleigh- and rainbow-like scattering, respectively. Of larger interest are any model regions showing iridescent-like phenomena.

The resulting single-scattering radiances at a fixed solar zenith angle and azimuth (values typical of the 9 January case 


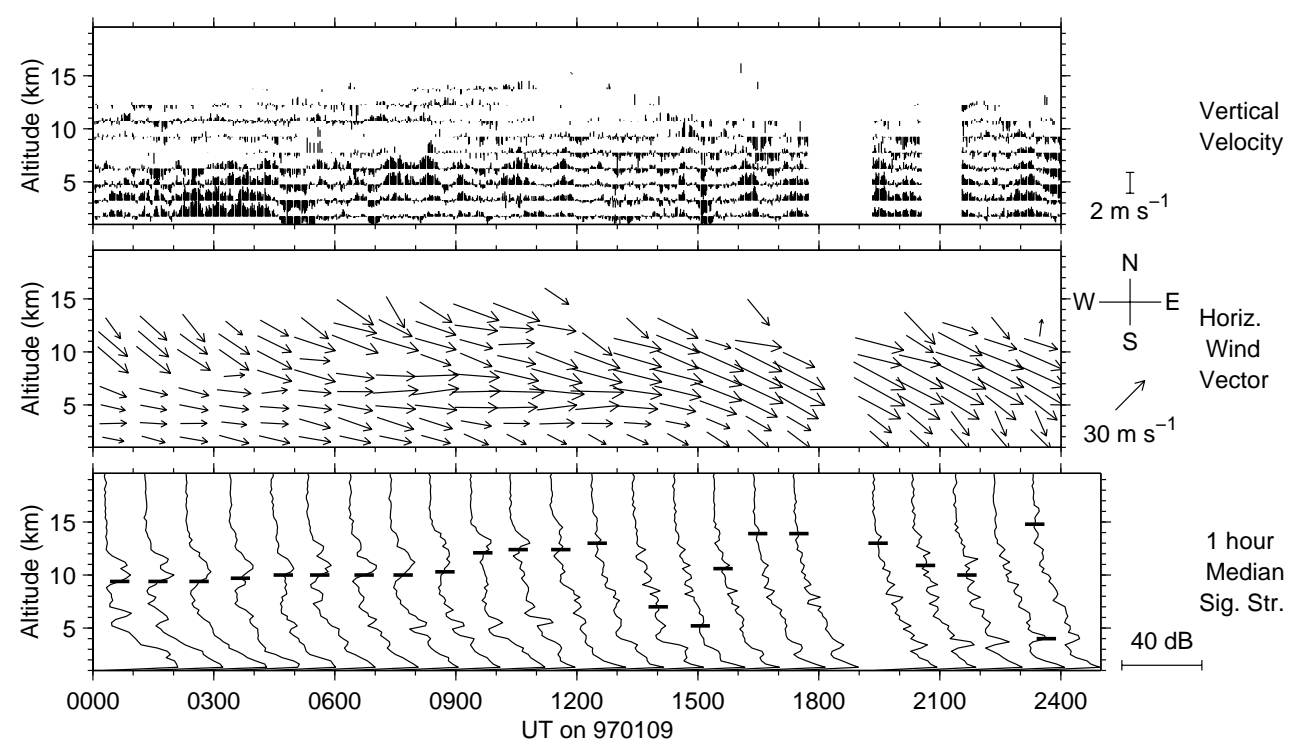

Fig. 5. ESRAD MST radar signal strengths with retrieved tropopause heights marked, horizontal and vertical winds on 9 January 1997.

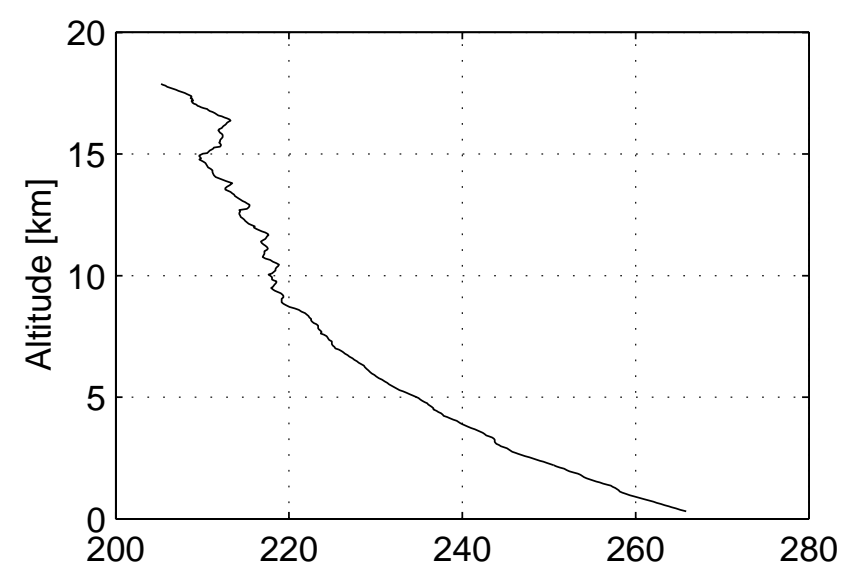

Fig. 6. Temperature profile from a radiosonde launched at Esrange on 9 January 1997.

below were used), summed over a number of wavelengths around $5590 \AA$ for three similar model volumes centred around different altitudes and superimposed on a smoothly varying background, have been projected to the image planes of station 1 (Fig. 3b) and station 3 (Fig. 3c). The resulting images resemble images of real mother-of-pearl clouds, at least in the backscatter geometry of station 1 . In the forward direction (station 3), however, oscillatory intensity structures appear, typical of the Mie solution, which are not visible in reality since PSCs must be optically thick to be visible. Nevertheless, the single-scattering approximation may be valid at least for some regions of PSC, e.g. when viewing the outer layers of PSCs such as the laminar "sandwich clouds" discussed by Biele et al. (2001) and Shibata et al. (1999).

The projections were divided into sub-blocks and for each projection altitude the correlation coefficient was calculated for each pair of blocks. The correlations varied rapidly with projection altitude, but at the (here a priori known) altitude of the "model PSCs" the curves corresponding to adjacent blocks follow each other well around their maxima. This is seen as a peak at $20 \mathrm{~km}$ in Fig. 4. As will be seen below, for our case of real PSC images the correlation has in fact a single maximum over the projection altitudes.

\section{Observations - the case of 9 January 1997}

Compared with previous years, an extreme number of visually observable PSC events occurred in January 1997. The polar vortex was strong and stable, leading to a synoptic PSC presence over large areas in the Arctic. Tropospheric winds were persistently strong and westerly, leading to additional mountain-wave perturbations. The 9th of January 1997 was chosen as a test case since there were well-defined discrete PSC structures north of Kiruna and the troposphere was clear. The PSCs were visible during daytime, observable as wavy mother-of-pearl clouds, and for the time after sunset when the images were taken.

The ESRAD MST radar (Fig. 5) showed a fluctuating vertical wind velocity, which is a signature of leewave activity, throughout the troposphere at the time of observation. Whether the waves propagated into the stratosphere is not evident (David Hooper, personal communication), but the presence of wave-like PSC structures can be interpreted as leewaves propagating into the stratosphere at least locally. The temperature profile of a radiosonde launched at Esrange the same day (Fig. 6) confirms this. Although the balloon burst at $18 \mathrm{~km}$, clear temperature fluctuations which may indicate wave activity and/or proximity to the vortex edge region did appear. 


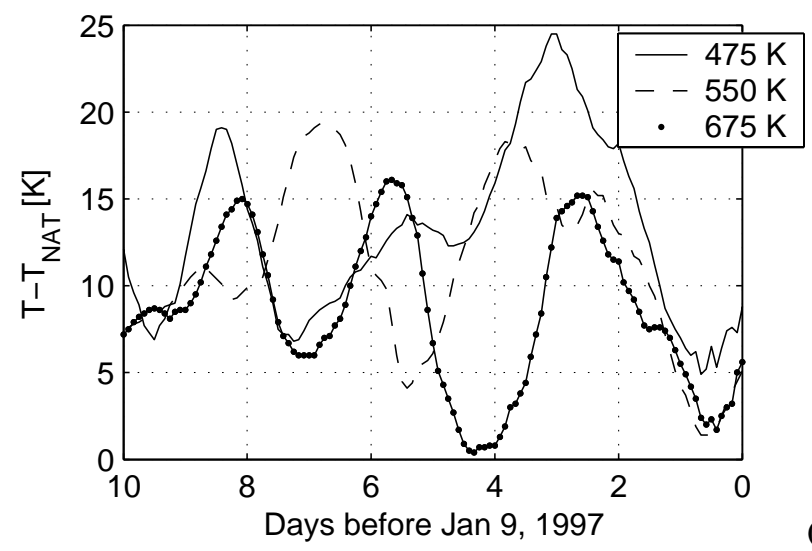

(a)

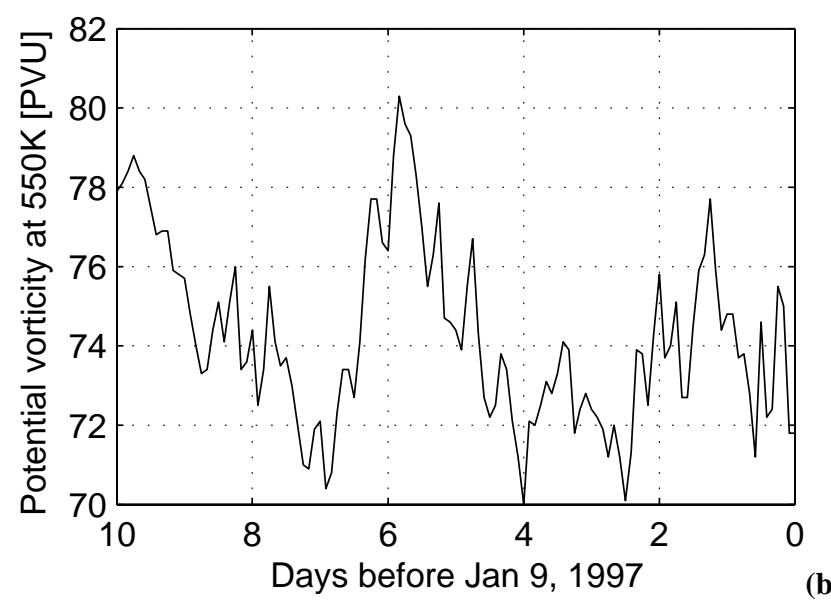

Fig. 7. Temperatures (a) and potential vorticity (b) along ECMWF backtrajectories from Kiruna for 9 January 1997.

\subsection{History of the observed air masses}

Synoptic-scale back-trajectories calculated from the ECMWF assimilated data (Fig.7a) indicated that the air mass arriving above Kiruna on 9 January 1997 had been repeatedly warmed and cooled to temperatures close to $T_{\mathrm{NAT}}$ for the 10 preceding days. The assumed values of $T_{\mathrm{NAT}}$, calculated according to Hanson and Mauersberger (1988) with $4.6 \mathrm{ppm}_{2} \mathrm{O}$, are given in Table 1, together with typical polar stratosphere altitudes of the isentropic levels. From the ECMWF analysis it is evident that the vortex edge was close to Kiruna and the values of potential vorticity (Fig. 7b) are also indicative of this fact.

Especially interesting is the fact that the air mass experienced a cooling followed by a slight warming during the last few days before 9 January 1997. The subsequent rapid cooling in the leewaves can drive the aerosol composition out of chemical equilibrium (Tsias et al., 1997). This fact is likely to account for the narrow particle size distribution required to produce the spatially quasi-fixed colour pattern which is so characteristic for leewave-induced PSC events like the present case.
Table 1. $T_{\text {NAT }}$ for the three isentropic levels under consideration calculated according to Hanson and Mauersberger (1988) with $4.6 \mathrm{ppm} \mathrm{H}_{2} \mathrm{O}$

\begin{tabular}{ccc}
\hline Level & $\begin{array}{c}\text { Appr. altitude } \\
{[\mathrm{km}]}\end{array}$ & $\begin{array}{c}T_{\mathrm{NAT}} \\
{[\mathrm{K}]}\end{array}$ \\
\hline $475 \mathrm{~K}$ & 20 & 194.5 \\
$550 \mathrm{~K}$ & 23 & 191.6 \\
$675 \mathrm{~K}$ & 26 & 187.8 \\
\hline
\end{tabular}

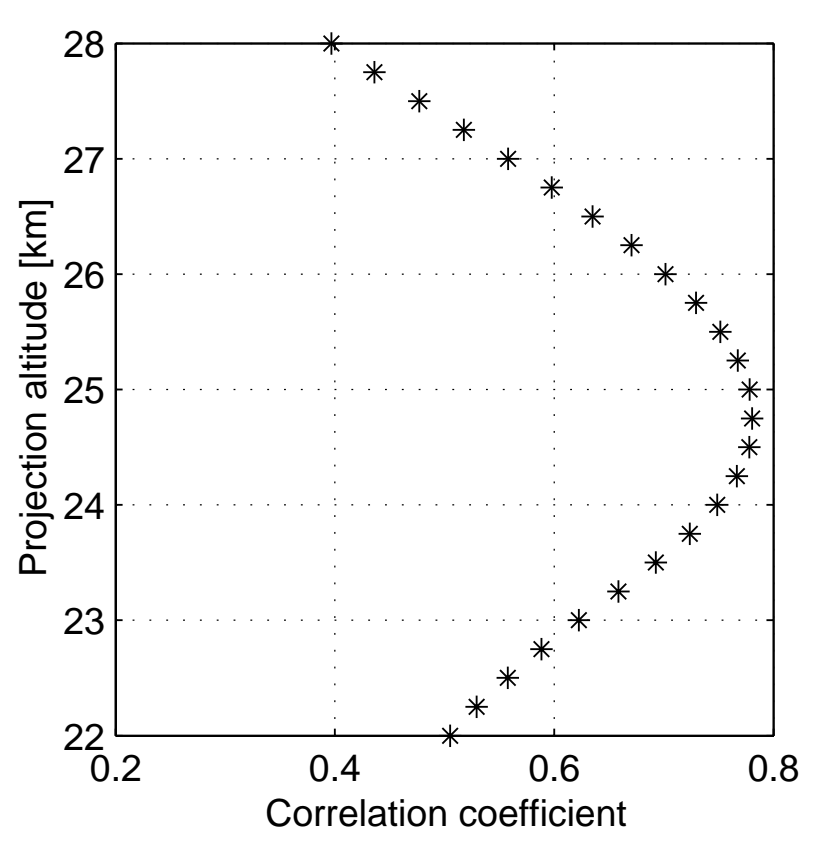

Fig. 8. Typical coefficients of 2-D spatial correlations of image pairs projected to consecutive altitudes.

\subsection{The bistatic image series}

A series of more than 20 image pairs was taken after sunset, between 14:00 and 16:00 UTC. Station 1 imaged in white light, whereas station 3 used the $5590 \AA$ filter to reduce the high sunset radiance. The exposure times used varied between $10 \mathrm{~ms}$ and $60 \mathrm{~s}$ depending on the time after sunset and on filtering.

The solar depression varied from 8 to 16 degrees during this time interval. The PSCs were therefore illuminated by scattered light only, since the daylight terminator was in fact well south of Scandinavia. The 9th of January 1997 was also a day of new moon, so additional lunar illumination can be ruled out. A smooth intensity variation is therefore observed, in contrast to the iridescense observed on direct solar illumination. The central parts of the PSCs were also sufficiently optically thick to prevent star observations during the projection calibration. This also contributes to a well-defined observable structure. 

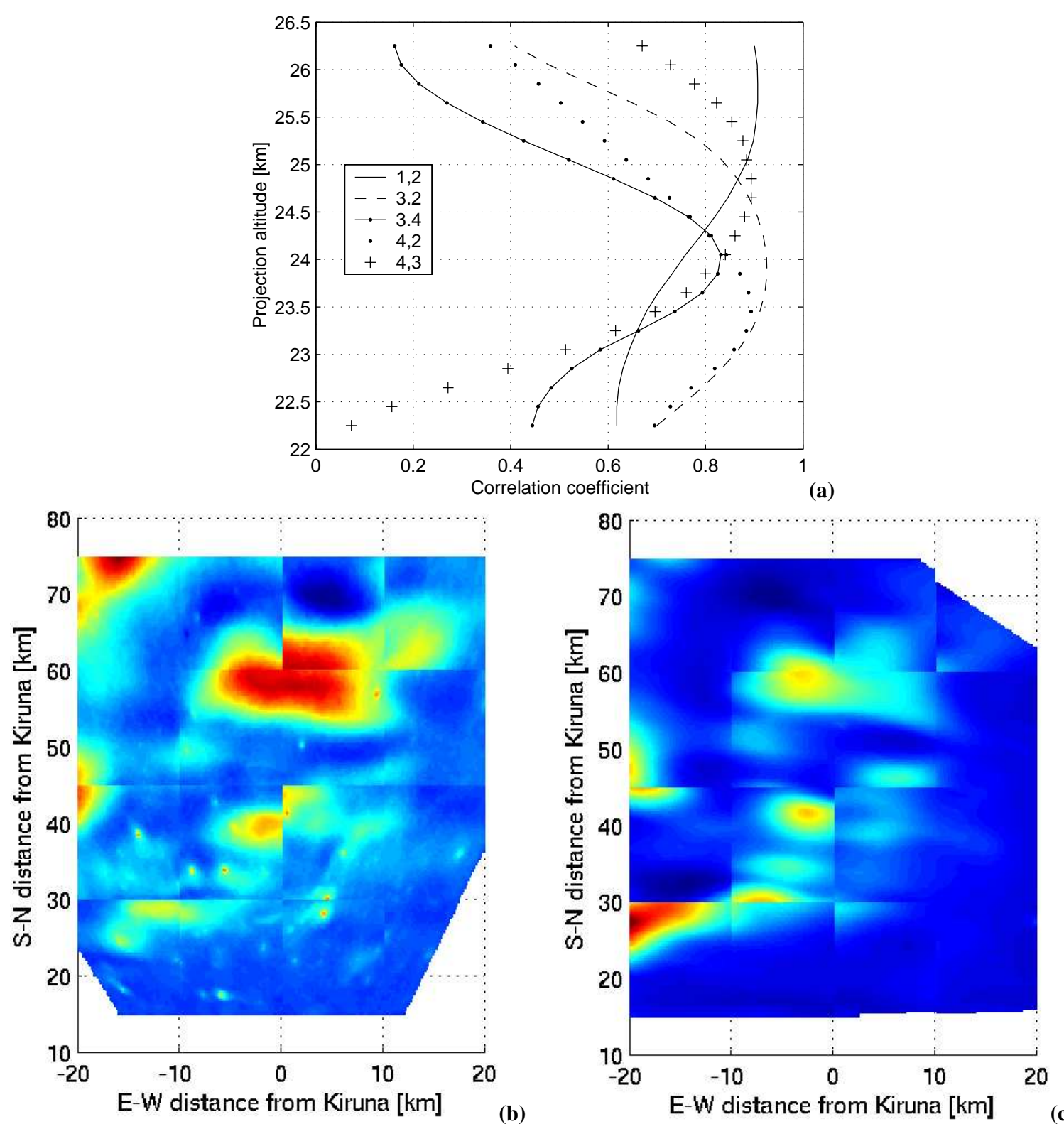

Fig. 9. Example of algorithm performance. (a): The spatial correlation vs. projection altitude for the PSC regions (sub-image row, column) of the images taken 15:21:30 UTC, and the corresponding projections from station 1 (b) and station 3 (c). The colour scale shows pixel values in arbitrary units.

\subsection{Automatic determination of equivalent altitude}

Correlation between the projections from stations 1 and 3 produced smooth curves (Fig. 8 is a typical result) in this case. The altitude of maximum correlation is therefore easily determined. On this day it varied around $25 \mathrm{~km}$, which is consistent with lidar observations (Reichardt et al., 1999), starting at 15:15 UTC at Esrange, located south of the observed PSCs between the stations, as shown in Fig. 1.

The same procedure was repeated with the projections divided into sub-blocks. In Fig. 9, the projections have been divided into $4 \times 4(256 \times 256$-pixel $)$ sub-images. This gives the possibility of sorting out only sub-images that actually contain PSC pixels. The sub-projections can also be allowed to have their maximal correlations at different altitudes, since wave clouds in general are not flat.

A problem of the block-wise projections is apparent, namely that the overlap is not smooth, especially for large blocks. Therefore we chose to use the equivalent altitudes obtained from the entire (non-divided) projections, since there were no tropospheric clouds in the present case. The time variation of the mean PSC altitudes thus obtained was examined with the Lomb-Scargle periodogram (Press et al., 1992). In Fig. 10 the result has been plotted (with the angu- 


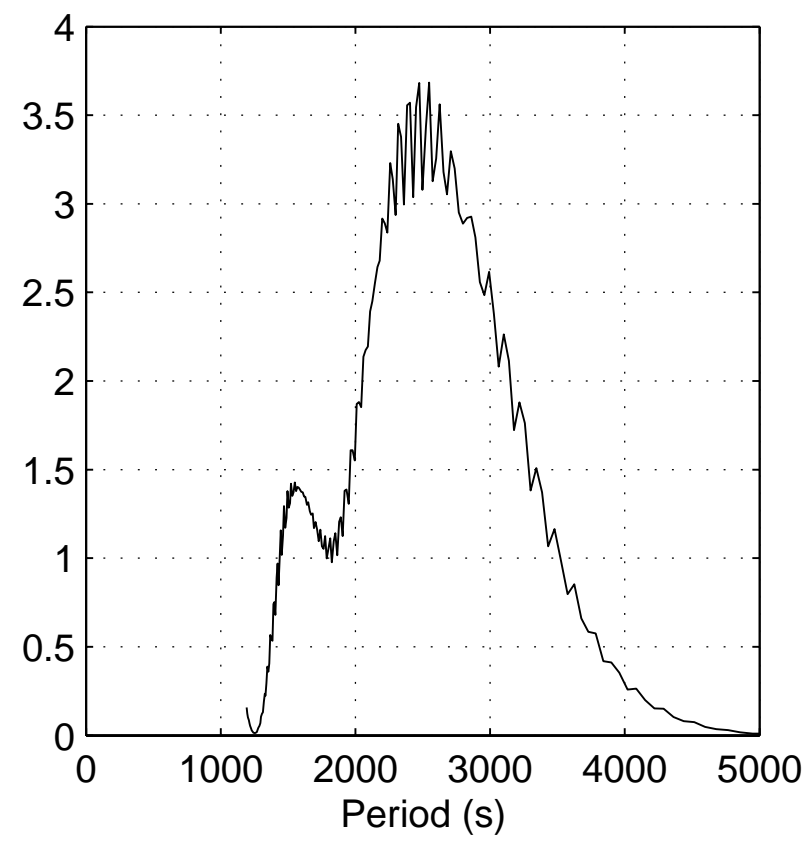

Fig. 10. Lomb-Scargle periodogram of the altitudes of maximal full-projection correlations for the image series of 9 January 1997. The abscissae are an interval of angular frequencies converted to periods.

lar frequency $\omega$ converted to period $T$ by $T=\frac{2 \pi}{\omega}$ ) over a range of periods. The main period present is approximately $40 \mathrm{~min}$. This may be due to a real period in the PSC altitude, connected with gravity wave activity, or to other effects (such as the aspect sensitivity caused by the fact that the PSC surfaces are sloping, whereas these projections are plane).

\subsection{Tracking features by manual triangulation}

To clarify the issue of altitude variation, manual triangulation of visible features of the PSCs was undertaken to track the 3-D surfaces spanned by those PSC points. These features are such edge points or contours which are clearly visible in both images in the bistatic image pairs. The lineof-sight separation will in the worst case correspond to an error in the retrieved altitude. For the present PSC geometry with its very low camera elevations this error reached $3 \mathrm{~km}(1 \sigma)$ during the first test runs. This error is of the same magnitude as the altitude variations retrieved below. This shows the importance of comparing independent retrieval algorithms and of monitoring the error display incorporated into the retrieval software meticulously during the identification of corresponding PSC features so that misidentified points can be discarded.

Figure 14, columns 1 and 2, shows a subset of these image pairs where correctly exposed images with distinct PSC features for triangulation have been selected. The points identified for triangulation are marked with corresponding numbers within each pair of images. The resulting PSC altitudes are also visualised. Column 3 shows projections of the im-
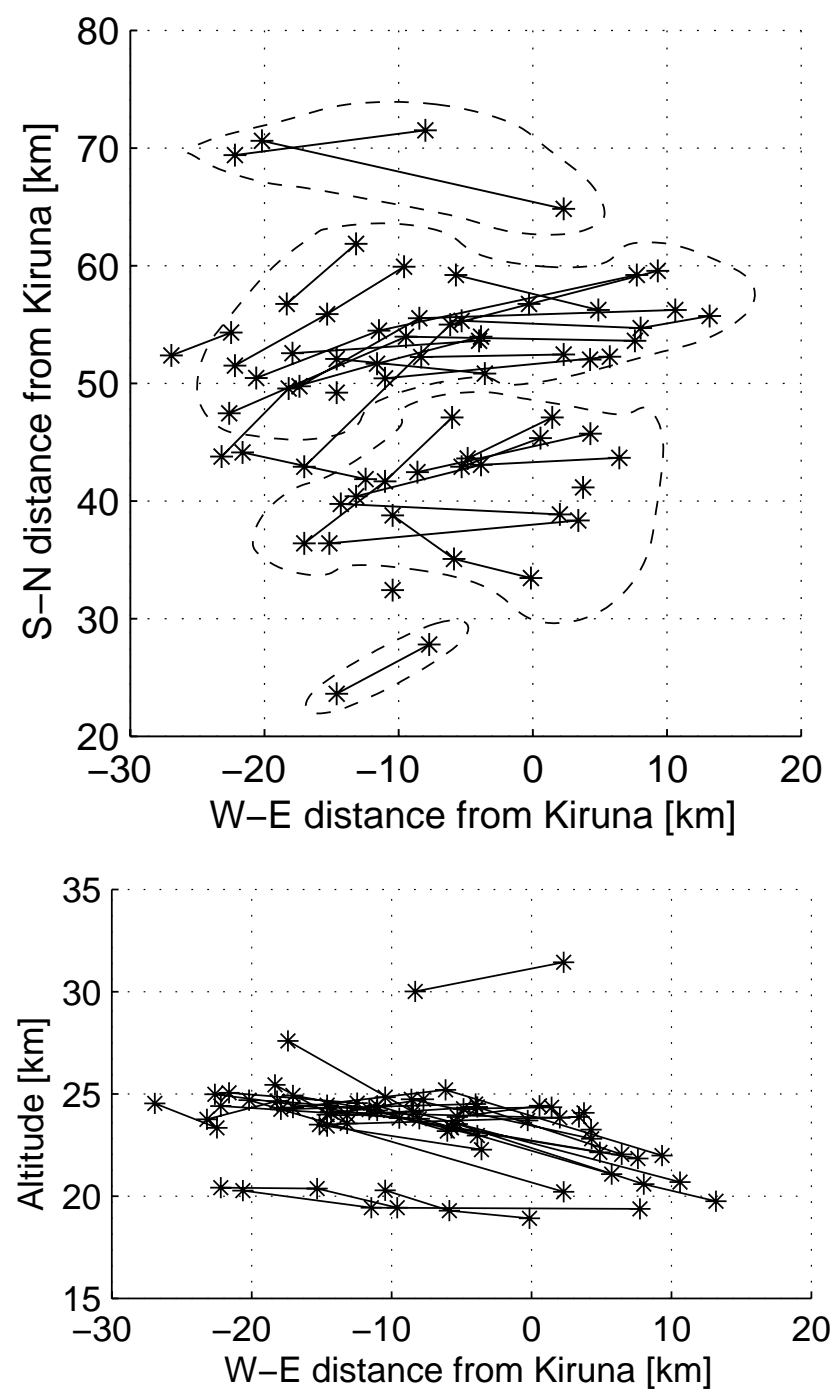

Fig. 11. Identifiable contour lines from triangulations using all image pairs with as many triangulation points as possible per pair. The points show the triangulated positions (as marked by red dots in Fig. 14); points belonging to the same visual contour features of the PSCs are joined by lines. These PSC "edges", and hence the PSCs, show a grouping in structures separated $10-20 \mathrm{~km}$. (a): W-E/N-S location; (b): altitude.

ages from station 1 (Kiruna) with superimposed contours of equal altitude. From column 3 it is evident that the retrieved altitude contours must not be interpreted as outlines of the PSCs but as surfaces containing spatially separated PSCs.

In Fig. 11, we concentrate on identified points in welldefined visual contour lines around the PSCs. Points within the same contour line are connected with lines. Although these points do not correspond to the same contours for all images, the result confirms the grouping of visible PSCs into discrete structures separated by $10-20 \mathrm{~km}$. Figure $11 \mathrm{~b}$, in which the points are viewed in the $X-Z$ plane, i.e. a westeast altitude transect, shows the persistent slope of the PSC field from 25 down to $20 \mathrm{~km}$ in the west-east direction.

The temporal order of the lines in Fig. 11 is not shown. 


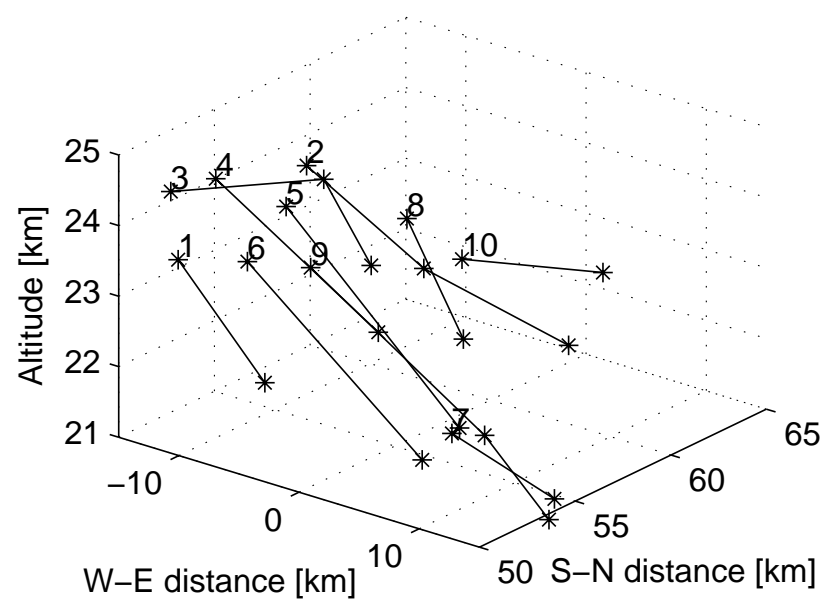

Fig. 12. Time development of a single visually identified feature in the PSC field observed on 9 January 1997. A minimum of two points in the contour have been identified in each image pair where possible. As above, distances are in $\mathrm{km}$ relative to the Kiruna station. The numbering refers to the same times of day as the abscissae of Fig. 13, in ascending order.

For this reason, one single PSC feature present in as many as possible of the best exposed image pairs has been singled out. The spatial locations of this single line during the time sequence is shown in Fig. 12. Lines some kilometres apart from the main grouping appear, which possibly should be removed as misidentified outliers. However the remaining results fall within the PSC field seen above, spatially sloping from an altitude above $25 \mathrm{~km}$ down to $20 \mathrm{~km}$. Therefore it seems that the altitude variation of the PSCs takes place on an isentrope distorted by mountain-wave activity. Figure 13a shows the time variation of the mean altitude of the lines. The time variation of the mean altitude is apparently quasi-periodic, as before. The Lomb-Scargle periodogram of these altitudes vs. time has been calculated in the same way as for Fig. 10 and is shown in Fig. 13b. Again, a period of close to $40 \mathrm{~min}$ appears, as in the automatic matching retrieval. The image series used covers only slightly more than an hour. However, the use of unevenly spaced data samples reduces the degeneracy so that substantially less aliasing of short-term variations occurs (Press et al., 1992). Therefore, we believe that this (quasi-)period can indeed be present in the altitude variation.

Since periodicity in leewave-PSC motion must be connected to the wave activity, it should be evident also in the observed vertical wind. The time period shown in Fig. 13 is in fact coincident with a wave of similar periodicity seen in the tropospheric wind speed in Fig. 5, upper panel. Also notable is the rapid drop of the retrieved radar tropopause shown in the lower panel due to these wave disturbances. Therefore the observed motion might be interpreted as a transient event rather than a period.
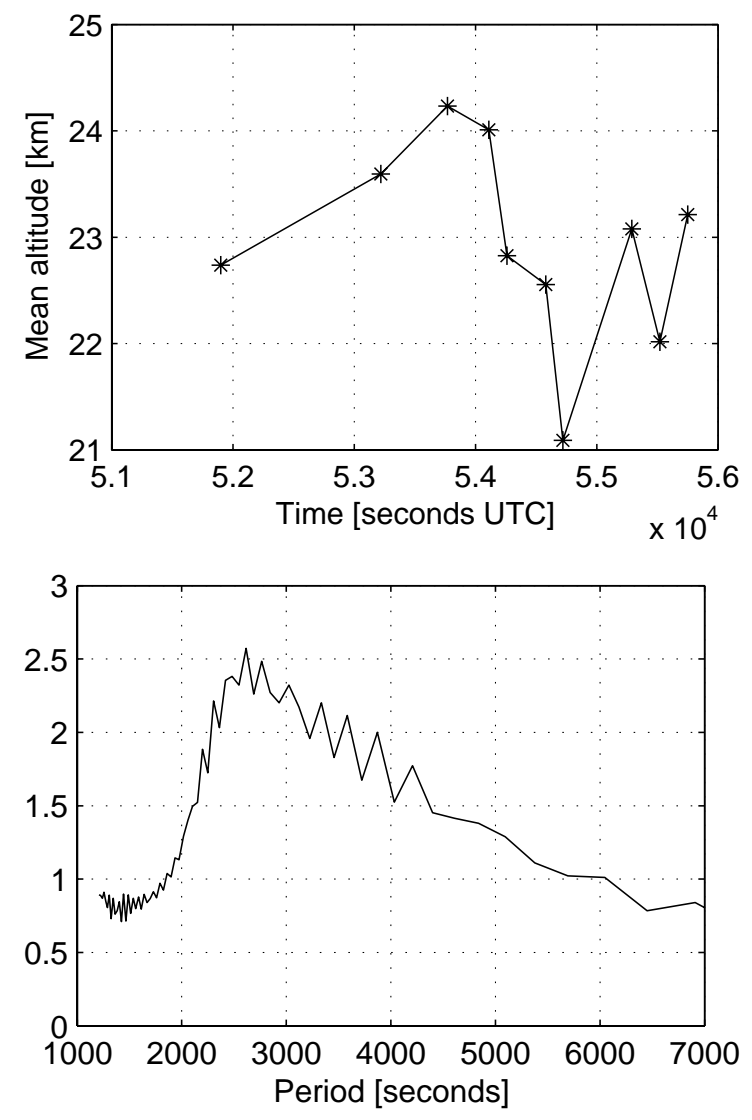

Fig. 13. (a): The mean altitude vs. time of day (here shown in seconds) of the lines in Fig. 12, 14:10:00 UTC (51000s) to 15:33:20 UTC (56000s). (b): Lomb-Scargle periodogram of the altitude variation.

\section{Conclusions}

We have observed PSCs during twilight on 9 January 1997. Discrete mother-of-pearl-like structures, also conspicuously visible during daytime, moved within a stationary sloping surface. This can be interpreted as fluctuations in a quasistationary wave pattern, since the PSCs can be assumed to form at the wave-induced temperature minima. Since the sun was below the horizon, the visual effects of a changing solar zenith angle should not lead to the misidentification of structures. The site of observation was at the edge of the polar vortex, and the air masses also originated from the vortex edge. Taken together, this provides some evidence that the observed PSCs were indeed leewave-induced. This is also in agreement with Hesstvedt (1962); Tsias et al. (1997) and other works, which draw the conclusion that a monodisperse particle size distribution is required for the iridescent colours observed earlier on the same day in the PSC field to appear, and that this is likely to be obtained under the conditions prevailing on 9 January 1997.

Given that visible PSC structures correspond to temperature minima, we can furthermore confirm that wave-induced temperature variations on a scale of $10-20 \mathrm{~km}$ occur. As pointed out by Carslaw et al. (1998b) this can cause a dis- 

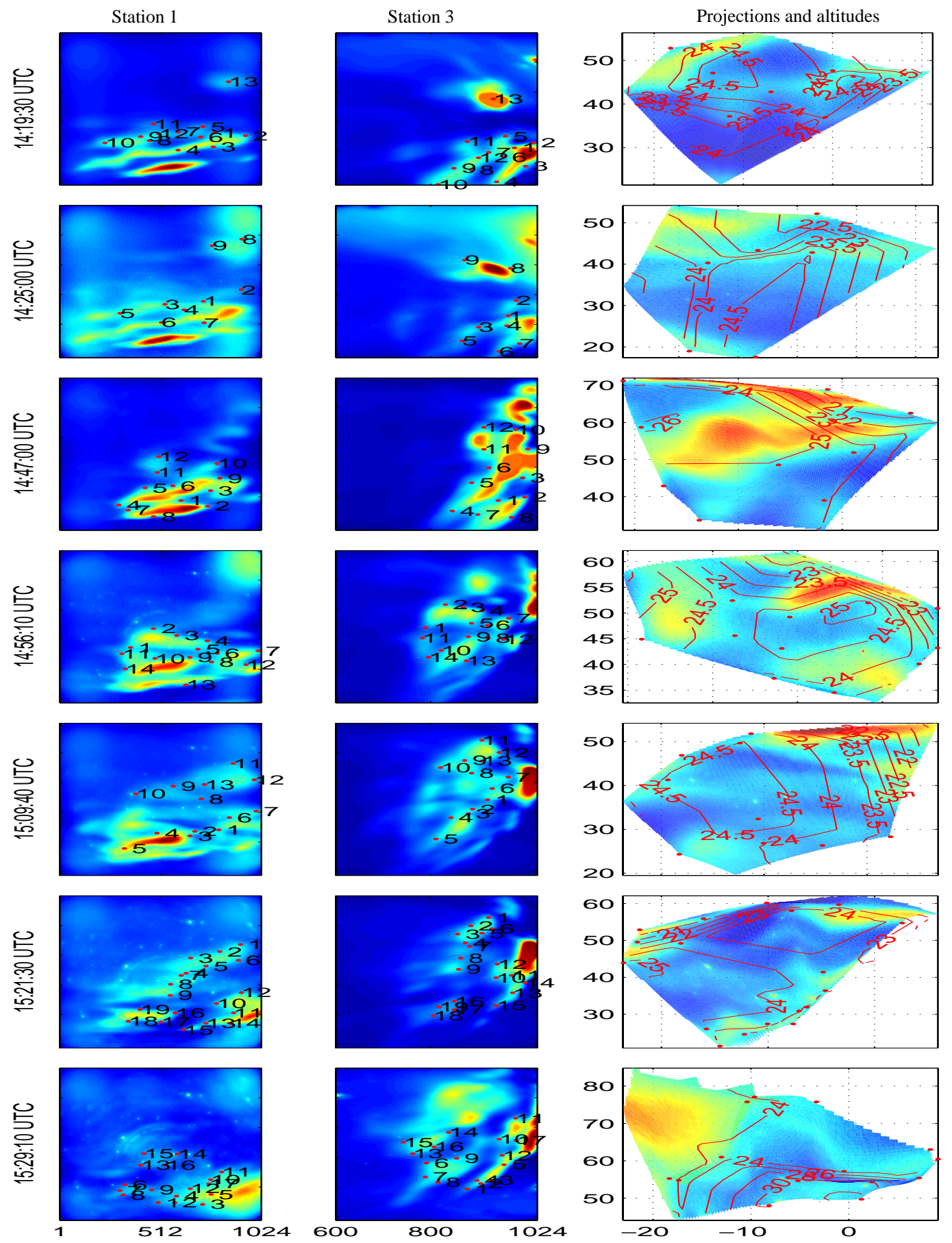

Fig. 14. Columns 1 and 2: Time series of images with identified PSC points (colour bars show the pixel values in $10^{4} \mathrm{~A} / \mathrm{D}$ counts after background subtraction). Rows 1-1024 and columns according to the abscissae are shown. Column 3: Contour surfaces (km) spanned by these points, superimposed on projections of the images from station 1. The axes show W-E and N-S distances in kilometres from Kiruna.

crepancy between observations and modelled values in studies of atmospheric chemistry. Until recently, it has been a time-consuming task to model such scales. At present, however, a comparison with temperature fields produced by mesoscale models is a viable task. The models should be tested for their ability to reproduce transient or quasi-periodic events perturbing gravity wave fields.
On 9 January 1997 only white-light and monochromatic imaging respectively were used at the two stations. For future work it would be desirable to repeat these observations with polarisation-sensitive devices, in order to characterise the scattered light with the full set of Stokes parameters at different wavelengths (Georg Witt, personal communication). Only this can yield information on particle shapes and the 
nature of the multiply scattered light illuminating the PSCs.

The possibility of imaging PSCs under different conditions of illumination, such as direct or scattered sunlight, moonlight, and even extinction of starlight, also deserves further experimental investigation.

Acknowledgements. Calculations of back-trajectories from ECMWF data, available at the Norwegian Institute of Air Research (NILU), have been provided by Bjørn Knudsen, Danish Meteorological Institute. The observations in January 1997 were performed within the framework of the European leewave project, using coordinated lidar, radar, and other observations in northern Scandinavia.

The work has been partly financed by the Environment and Space Research Institute in Kiruna (MRI).

The main author is also thankful to Peter Rydesäter for many discussions on image processing.

Topical Editor U.-P. Hoppe thanks two referees for their help in evaluating this paper.

\section{References}

Biele, J., Tsias, A., Luo, B., Carslaw, K., Neuber, R., Beyerle, G., and Peter, T.: Nonequilibrium coexistence of solid and liquid particles in Arctic stratospheric clouds, J. Geophys. Res., 106, 22 991-23 007, 2001.

Bohren, C. F. and Huffman, D. R.: Absorption and scattering of light by small particles, John Wiley and Sons, Inc., New York, 1983.

Brändström, U. and Steen, Å.: ALIS - a new ground-based facility for auroral imaging in northern Scandinavia, in: Proc. 11th Symposium on European Rocket and Balloon Programmes and related research, pp. 301-306, Eurpoean Space Agency, ESA SP355, 1994.

Carslaw, K. S., Wirth, M., Tsias, A., Luo, B. P., Dörnbrack, A., Leutbecher, M., Volkert, H., Renger, W., Bacmeister, J. T., and Peter, T.: Particle microphysics and chemistry in remotely observed mountain polar stratospheric clouds, J. Geophys. Res., 103, 5785-5796, 1998a.

Carslaw, K. S., Wirth, M., Tsias, A., Luo, B. P., Dörnbrack, A., Leutbecher, M., Volkert, H., Renger, W., Bacmeister, J. T.,
Reimers, E., and Peter, T.: Increased stratospheric ozone depletion due to mountain-induced atmospheric waves, Nature, 391, 675-678, 1998b.

Dörnbrack, A., Leutbecher, M., Reichardt, J., Behrendt, A., Müller, K.-P., and Baumgarten, G.: Relevance of mountain waves for the formation of polar stratospheric clouds over Scandinavia: Mesoscale dynamics and observations for January 1997, J. Geophys. Res., 106, No. D2, 1569-1582, 2001.

Godin, S., Mégie, G., David, C., Haner, D., Flesia, C., and Emery, Y.: Airborne lidar observation of mountain-wave-induced polar stratospheric clouds during EASOE, Geophys. Res. Lett., 21, 1335-1338, 1994.

Gustavsson, B.: Three Dimensional Imaging of Aurora and Airglow, Ph.D. thesis, Swedish Institute of Space Physics, ISBN 91-7191-878-7, 2000.

Hanson, D. and Mauersberger, K.: Laboratory studies of the nitric acid trihydrate: Implications for the south polar stratosphere, Geophys. Res. Lett., 15, 855-858, 1988.

Hesstvedt, E.: A two-dimensional model of mother-of-pearl clouds, Tellus, XIV, 297-300, 1962.

Peter, T.: Microphysics and heterogeneous chemistry of polar stratospheric clouds, Annual Review of Physical Chemistry, 48, 785-822, 1997.

Press, W. H., Teukolsky, S. A., Vetterling, W. T., and Flannery, B. P.: Numerical Recipes in C, Cambridge University Press, second edn., 1992.

Reichardt, J., Behrendt, A., Baumgart, R., and Weitkamp, C.: Koordinierte Feldmessungen zum Einfluß von Leewellen auf Wolkenfelder in der polaren Stratosphäre, Tech. Rep. GKSS 99/E/43, GKSS Forschungszentrum, Geesthacht, Germany, 1999.

Shibata, T., Shiraishi, K., Adachi, H., Iwasaka, Y., and Fujiwara, M.: On the lidar-observed sandwich structure of polar stratospheric clouds (PSCs) 1. Implications for the mixing state of the PSC particles, J. Geophys. Res., 104, 21 603-21 611, 1999.

Steen, Å., Gustavsson, B., and Brändström, U.: Temporal variation of 2-D altitude distribution of lee-wave generated polar stratospheric clouds, in: Polar stratospheric ozone 1997, Proceedings of the fourth European symposium, Air pollution research report 66, European Commission, 1998.

Tsias, A., Prenni, A. J., Carslaw, K. S., Onasch, T. P., Luo, B. P., Tolbert, M. A., and Peter, T.: Freezing of polar stratospheric clouds in orographically induced strong warming events, Geophys. Res. Lett., 24, 2303-2306, 1997. 